\title{
The influence of the strength of bone on the deformation of acetabular shells
}

\section{A LABORATORY EXPERIMENT IN CADAVERS}

\author{
M. C. Bone, P. Dold, M. Flohr, R. Preuss, T.J. Joyce, R.M. Aspden, J. Holland, D. Deehan
}

From Newcastle Biomedical Research Centre, Newcastle, United Kingdom

M.C. Bone, BEng, Research Assistant, School of Mechanical and Systems Engineering

T. J. Joyce, PhD, Professor of Orthopaedic Engineering, School of Mechanical and Systems

Engineering Newcastle University, Stephenson Building, Claremont Road, Tyne and Wear NE1 7RU, UK.

P. Dold, M.Eng, Development Engineer, Medical Products Division

M. Flohr, Dipl.-Ing, Manager Product Testing and Biomechanics

R. Preuss, Dr.-Ing, VP R\&D/ Reg.Aff CeramTec GmbH, 73207 Plochingen, Germany.

R. M. Aspden, FIPEM, DSc, PhD, Professor in Orthopaedic Science University of Aberdeen, IMS

Building, Foresterhill, Aberdeen AB25 2ZD, UK.

J. Holland, FRCS (Orth), Orthopaedic Consultant

D. Deehan, MD FRCS (Tr \& Orth), Professor Freeman Hospital, Newcastle Upon Tyne NE7 7AH, UK.

Correspondence should be sent to Mr M. C. Bone; e-mail: mbone05@hotmail.co.uk 


\begin{abstract}
Concerns have been raised that deformation of acetabular shells may disrupt the assembly process of modular prostheses. In this study we aimed to examine the effect that the strength of bone has on the amount of deformation of the acetabular shell. The hypothesis was that stronger bone would result in greater deformation. A total of 17 acetabular shells were inserted into the acetabula of eight cadavers, and deformation was measured using an optical measuring system. Cores of bone from the femoral head were taken from each cadaver and compressed using a materials testing machine. The highest peak modulus and yield stress for each cadaver were used to represent the strength of the bone and compared with the values for the deformation and the surgeon's subjective assessment of the hardness of the bone. The mean deformation of the shell was $129 \mu \mathrm{m}$ (3 to 340). No correlation was found between deformation and either the maximum peak modulus $\left(r^{2}=0.011, t=0.426, p=0.676\right)$ or the yield stress $\left(\mathrm{r}^{2}=0.024, \mathrm{t}=0.614, \mathrm{p}=0.549\right)$ of the bone. Although no correlation was found between the strength of the bone and deformation, the values for the deformation observed could be sufficient to disrupt the assembly process of modular acetabular components.
\end{abstract}


During the 1970s uncemented acetabular components became popular, despite more than a decade of success using cemented components.[[1]] Uncemented components remain popular [Jameson et al. 2013]; data from the 2013 National Joint Registry of England, Wales and Northern Ireland showed that between 2004 and 2012 the use of cemented acetabular components declined.[[2]] Cementless acetabular components require initial stability through a press-fit within the acetabulum.[[3,4]] Although uncemented components offer advantages such as preservation of bone stock,[[5]] there are concerns that the forces required to achieve initial fixation may result in significant deformation of the acetabular shell.[[6-8]]

Deformation is thought to be linked to several variables. Previous in-vitro, cadaveric and finite element studies have examined deformation of the acetabular component with regard to different variables such as the diameter of the component,[[4,9]] underreaming,[[6]] the thickness of the component[[9,10]] and positioning.[[11]] We are not aware of studies that have related deformation the strength of the bone.

The aim of this study in cadavers was to determine whether deformation of the acetabular shell correlates with the stiffness or strength of the underlying bone. The hypothesis was that stiffer and/or stronger bone would result in greater deformation.

\section{Materials and Methods}

\section{Cadaver and implant details}

A series of 17 titanium acetabular shells (each used only once) were inserted into eight cadavers in a temperature-controlled laboratory. The posterior surface of the shells was grit blasted with an estimated roughness average of 2 to $5 \mu \mathrm{m}$. There were three female and five male cadavers. Their mean age at the time of death was 74.1 years (58 to 87); their mean weight was $72.4 \mathrm{~kg}$ (32 to 113) and their mean height was $170 \mathrm{cms}$ (152 to 193). The shells were custom manufactured for these experiments by CeramTec (CeramTec GmbH, Plochingen, Germany) from a titanium alloy (TiAl6V4) with a thickness of $3 \mathrm{~mm}$. As the 
sizes of the cadaver acetabula could not be determined prior to the start of the experiment, a range of external diameters of the shell between $44 \mathrm{~mm}$ and $62 \mathrm{~mm}$ were available.

Table 1. Details of the cadavers

\begin{tabular}{|l|l|l|l|l|}
\hline Cadaver & Gender & Age (yrs) & Height (cm) & Weight (kg) \\
\hline $\mathbf{1}$ & Female & 72 & 170 & 43 \\
\hline $\mathbf{2}$ & Male & 70 & 168 & 73 \\
\hline $\mathbf{3}$ & Male & 80 & 193 & 80 \\
\hline $\mathbf{4}$ & Female & 82 & 152 & 32 \\
\hline $\mathbf{5}$ & Male & 58 & 175 & 113 \\
\hline $\mathbf{6}$ & Male & 85 & 163 & 84 \\
\hline $\mathbf{7}$ & Female & 59 & 163 & 68 \\
\hline $\mathbf{8}$ & Male & 87 & 178 & 86 \\
\hline
\end{tabular}

\section{Surgical method}

A large incision was created using a posterior approach and up to $20 \mathrm{~cm}$ of the proximal femur was resected in order to provide the necessary access for the measurement system. Reaming was performed in $2 \mathrm{~mm}$ increments until the true floor of the acetabulum was exposed. Next, the reaming was expanded to remove the cartilage down to a hemispherical subchondral bed of bone suitable to securely press-fit a hemispherical shell without the need for screw fixation. After reaming, the surgeon graded the bone on a 3-point scale, 1 being the hardest and 3 the softest. The shells were then implanted as intended in a standard hip replacement. The amount of reaming was adjusted to achieve stable primary fixation that would be acceptable in live surgery in all cases. This involved reaming "to size" in eight shells, and under-reaming by $1 \mathrm{~mm}$ in nine shells. Fixation was defined as complete medial contact with the surgeon unable to pull the shell out or rotate it manually. In order to maximise the usefulness of the cadaver, in large patients it was sometimes possible to re- 
ream the acetabulum and insert a larger shell while still obtaining a secure press-fit. Shells were therefore inserted into 11 acetabula, with five acetabula having more than one implanted.

\section{Measurement of Deformation}

Measurements were performed immediately before and within five minutes after introduction of the shell using the GOM ATOS III Triple Scan optical system (GOM GmbH, Braunshweig, Germany). These two measurements were then compared to determine the deformation in a plane at $1 \mathrm{~mm}$ below the rim of the shell. This distance was chosen because previous measurements using this system have shown that although the shell is subject to asymmetrical deformation, the highest values are recorded at the rim.[[12]] The GOM system uses two cameras to determine the position and size of the object using an interferometric technique. A previous validation study [[12]] found that the maximum difference between the GOM system and a coordinate measuring machine (CMM) (Carl Zeiss Industrielle Messtechnik GmbH, Oberkochen, Germany) for this application was $5 \mu \mathrm{m}$. A thin titanium oxide $\left(\mathrm{TiO}_{2}\right)$ coating was applied to the internal surfaces of the shells to dull the reflective surface. In order to prevent damage to the coating, surgical swabs were applied to the area surrounding the shell to absorb any moisture. The method of measurement and validation have been described previously.[[13]]

\section{Testing the strength of bone}

Core samples of cancellous bone were taken from each cadaver following the method described by Li and Aspden[[14]], to examine the mechanical properties of femoral cancellous bone. A total of 75 samples were taken from five sites across each of the 15 femoral heads: the superior, inferior, anterior, posterior and central surfaces. No samples were taken from one femoral head as no shells had been implanted in the corresponding 
acetabulum. Cores were removed using a bit and drill approximately perpendicular to the surface, so that the axis of the cylinder was towards the centre of the femoral head.

For each sample, the subchondral bone was removed and the ends were trimmed. The mean diameter of the core was $7.8 \mathrm{~mm}$ (7.2 to 8.2) and the mean length was $12.7 \mathrm{~mm}$ (6.7 to 21.0). Each sample was then subjected to an unconstrained compression test on an Instron 5564 materials testing machine (Instron, High Wycombe, Buckinghamshure, United Kingdom) using the technique described previously.[[14]] A strain rate of 20\% per minute was used and load-displacement curves were plotted. In order to avoid damaging the samples unnecessarily, tests were stopped manually once the slope of the curve was seen to be reducing.

\section{Data processing}

Loads and displacements were converted to stresses and strains by dividing by the original cross-sectional area and length of each sample. A program was written in MathCad (Parametric Technology Corporation, Needham, MA, USA) to import and analyse the stressstrain data. A polynomial curve was fitted to a section of the rising part of the curve and differentiated to determine the gradient. The region of the curve and the degree of polynomial (most commonly five) were selected to ensure close fitting to the steepest part of the curve without over-fitting, as judged visually and by least-squares distance between the fitted curve and the data. Key points of the test are shown in Figure 1. Peak modulus and yield stress were then obtained from the data. Peak modulus, and the corresponding stress and strain values, was taken as the greatest value of the gradient. Yield stress was the stress at the yield point, which was defined as the point at which the modulus decreased by $3 \%$ from its peak value. This may be too stringent, but uses the same approach as Li and Aspden.[[14]] The peak modulus is defined as the resistance of the material to the applied stress. Yield stress is the 
point at which elastic deformation stops and the sample is left with permanent or plastic deformation, once the load has been removed.[[15]]

Figure 1: A load extension graph for 3-point bending, however the principles are the same for stress strain tests

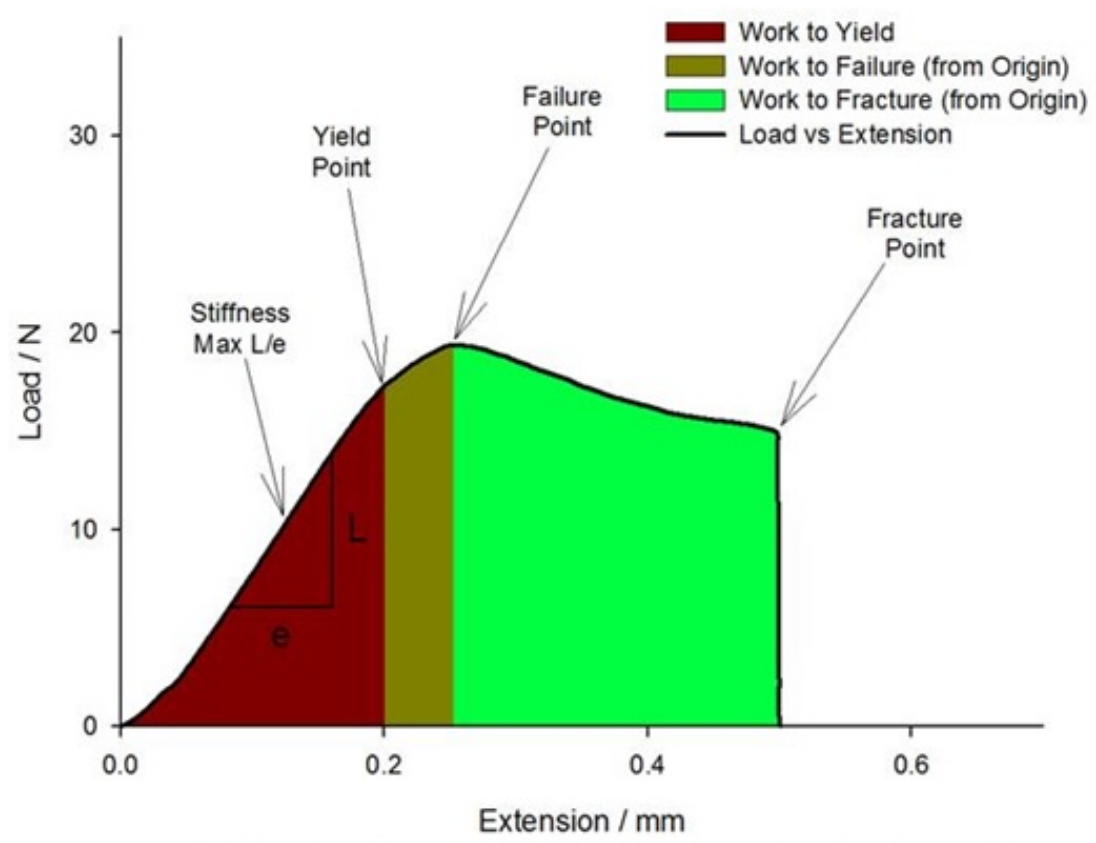

The greatest peak modulus and yield stress for each femoral head were recorded. Results from the left and right femora of the same cadaver were combined and the values for the overall greatest peak modulus and yield stress were used for that cadaver. These values were then compared with both the values for the deformation and the surgeon's grading of the bone. Although this provided information on how surgical 'feel' matches against mechanical data, it is acknowledged that the surgical grading is very subjective. It should be noted that femoral bone quality was taken as a surrogate marker for acetabular bone quality. 


\section{Statistical analysis}

The statistical significance of the relationships between deformation and both peak modulus and yield stress was assessed using the statistical package R (R Foundation for statistical computing, Vienna, Austria) and a linear regression. Deformation was assessed against the diameter of the shell using generalised linear models with a gaussian error structure to ensure that it did not influence the results, and $\mathrm{p}<0.05$ was considered to be statistically significant.

\section{Results}

\section{The strength of the bone}

During compression testing, data from the bone cores of one femur were lost owing to a computer malfunction, and 13 samples toppled over during testing when loaded, and so were excluded from the results. Data were therefore available from 57 cores. A minimum of two results for each femoral head and five results per cadaver were available for analysis. The mean peak modulus was $320.5 \mathrm{MPa}(79.6$ to 469.7$)$ and the mean yield stress was $6.5 \mathrm{MPa}$ (1.3 to 13.0).

Comparisons of the surgeon's grading of the bone and mechanical testing results are shown in Figures 2 and 3, respectively. The mean peak modulus for the eight cadavers did not match with the surgeon's grading, the grading being correct for only two samples (cadavers 1 and 4). Yield stress provided a closer agreement with the surgeon's grading than peak modulus, with the grading correct for five samples. 
Figure 2: Comparison of peak modulus and surgeon intra-operative grading ( 3 is the softest and 1 is the hardest)

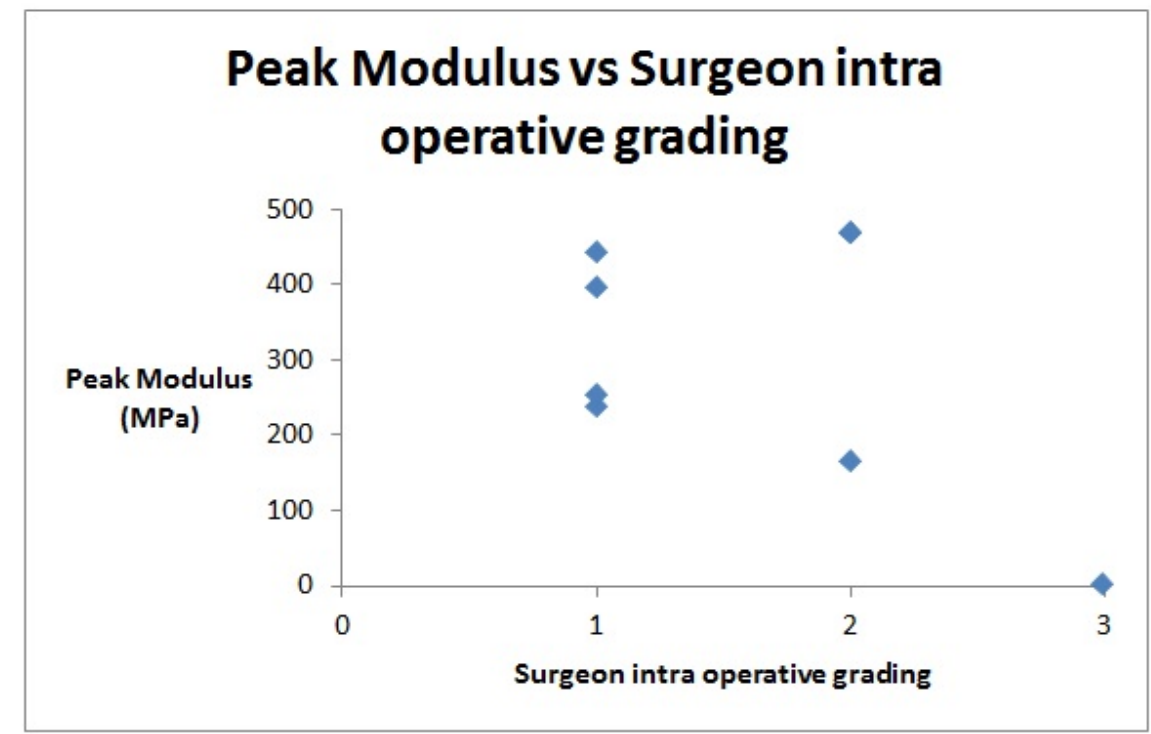

Figure 3: Comparison of yield stress and surgeon intra-operative grading ( 3 is the softest and 1 is the hardest)

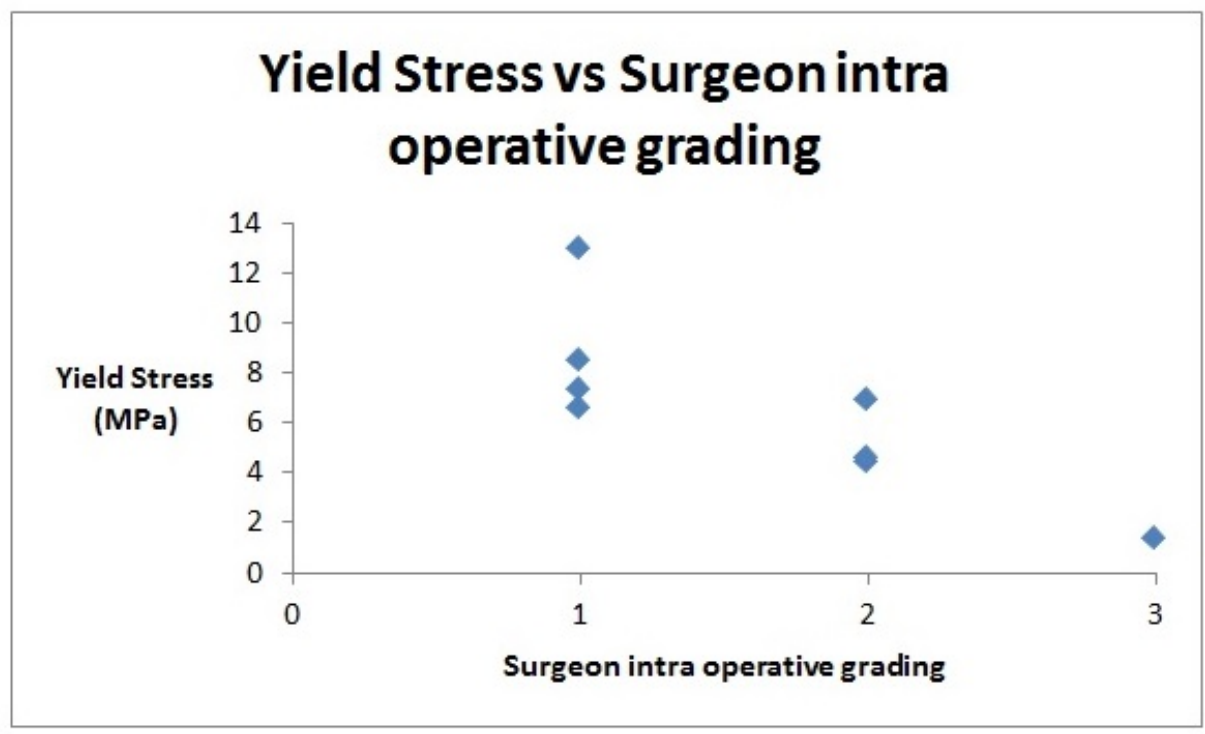

\section{Deformation}

Figure 4 shows the comparison between deformation of the shell and peak modulus, and

Figure 5 shows the comparison between deformation and yield stress for the 17 shells. The mean deformation was $129 \mu$ m (3 to 340). Figure 6 shows the deformation as a percentage 
change in diameter, and Figure 7 shows the comparison between deformation and the diameter of the shell.

Figure 4: Graph of peak modulus against deformation

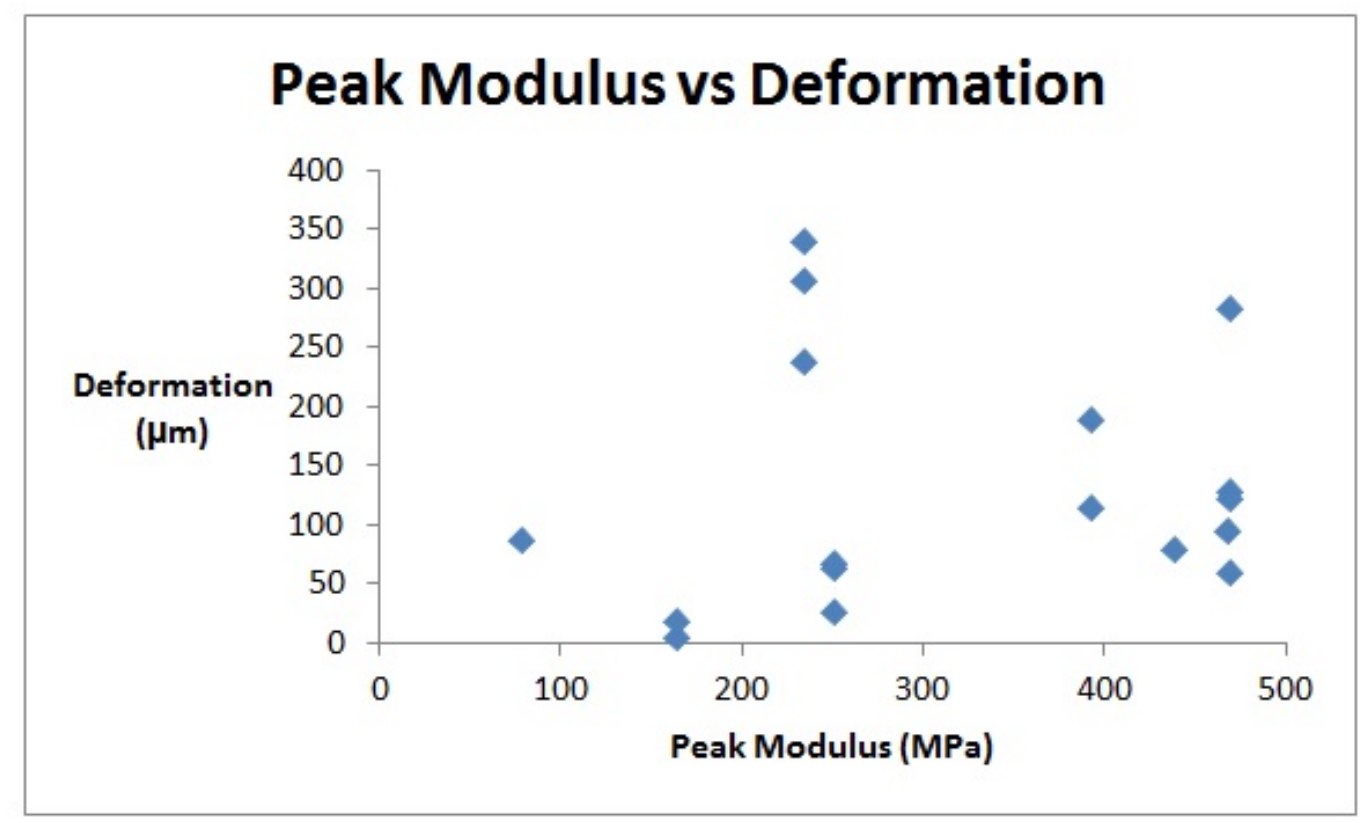

Figure 5: Graph of yield stress against deformation

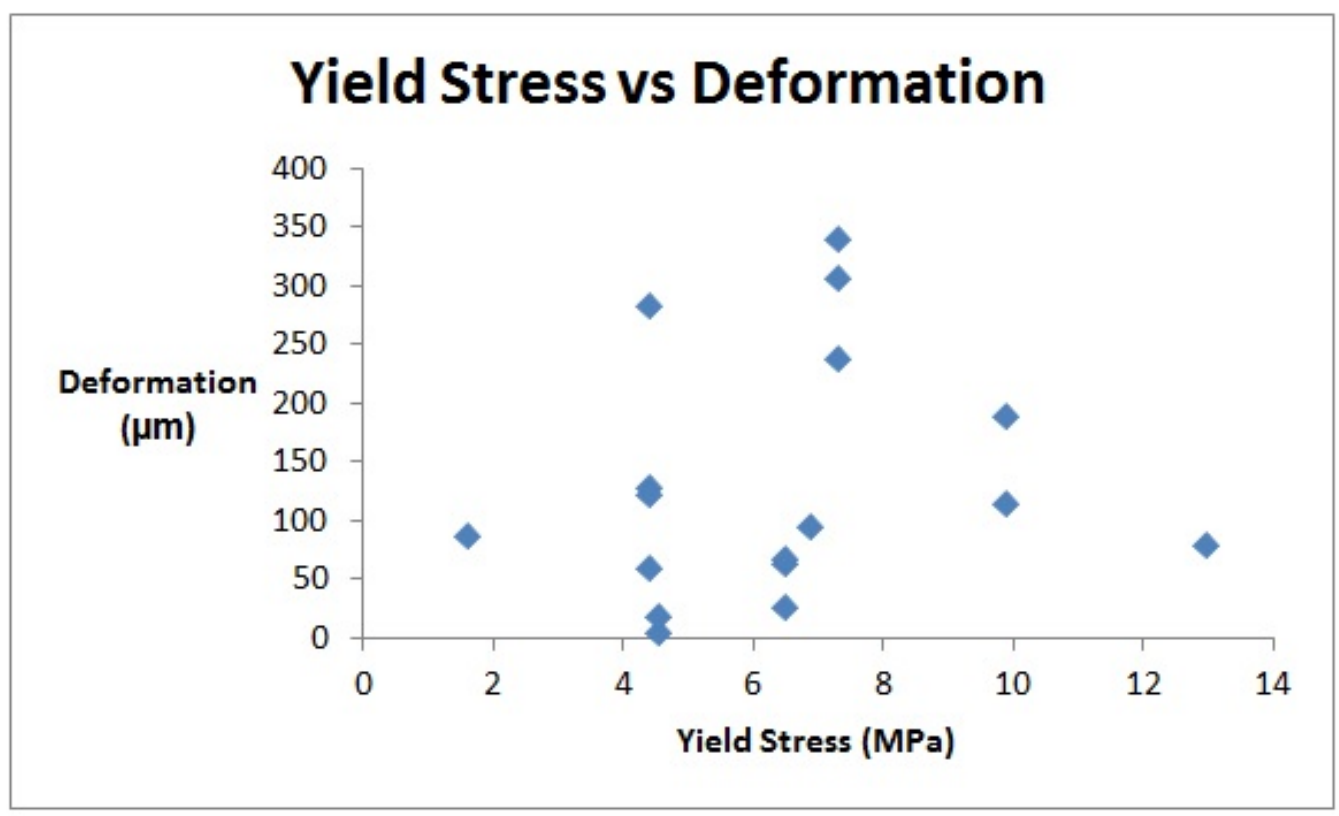


Figure 6: Graph of percentage change in diameter vs deformation

\section{Percentage change in diameter vs Deformation}

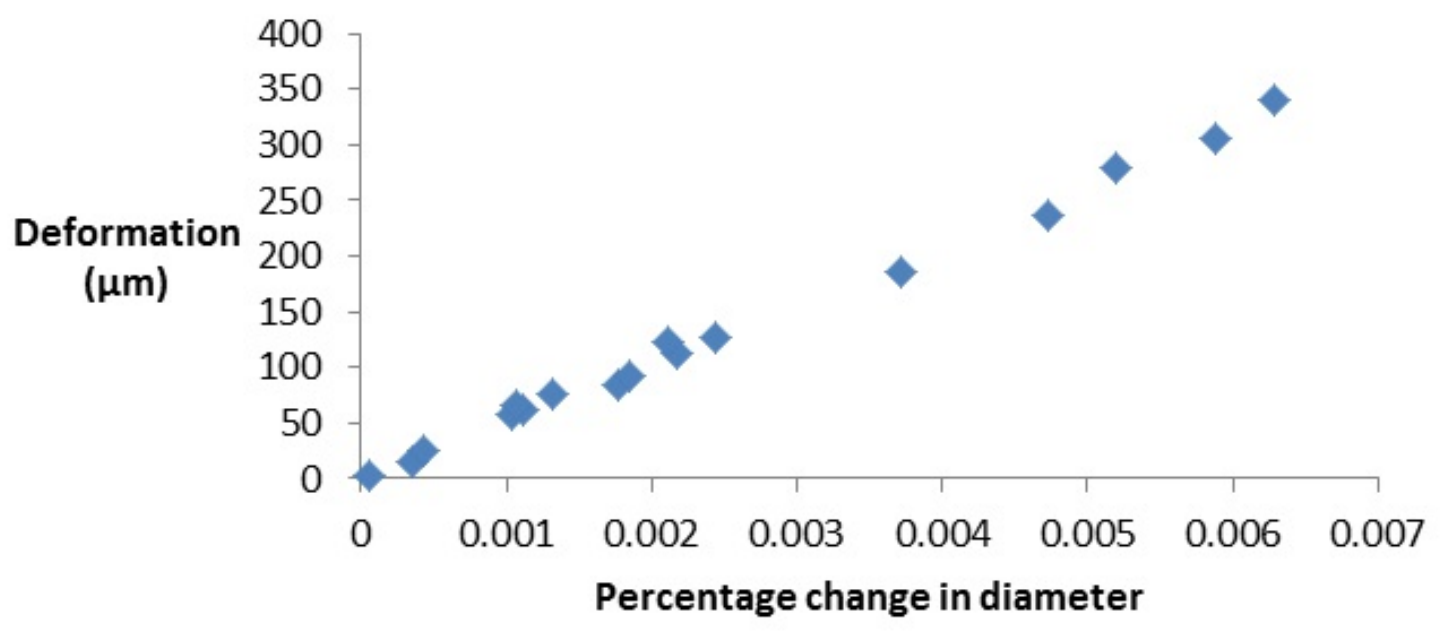

Figure 7: Graph of shell diameter against deformation

\section{Shell Diameter vs Deformation}

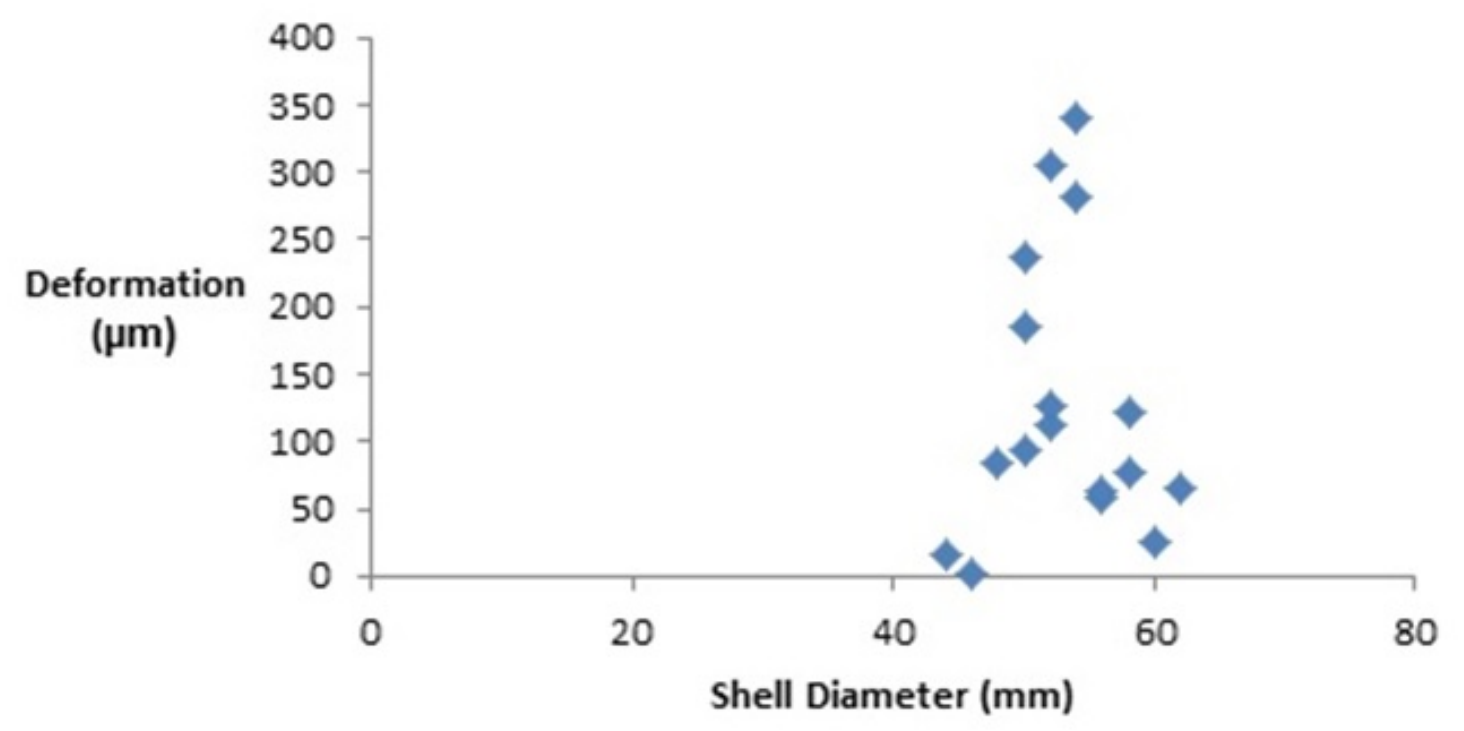

No correlation was found between deformation and peak modulus $\left(\mathrm{r}^{2}=0.012, \mathrm{t}=0.426, \mathrm{p}=\right.$ $0.676)$ or deformation and yield stress $\left(\mathrm{r}^{2}=0.024, \mathrm{t}=0.614, \mathrm{p}=0.549\right)$, nor was any 
correlation found between deformation and the diameter of the shell $\left(\mathrm{r}^{2}=0.000, \mathrm{t}=-0.026\right.$, $\mathrm{p}$ $=0.979)$.

\section{Discussion}

The lack of correlation between deformation and peak modulus or yield stress was in contrast to the hypothesis that greater strength or stiffness of bone would result in greater deformation.

Deformation has been linked with many variables but we believe this to be the first paper to examine the influence that bone has on deformation. The mean deformation of 129 $\mu \mathrm{m}$ found in our study was comparable with that reported previously. In 2006 Squire et al[[4]] reported a mean deformation of $160 \mu \mathrm{m}$ (0 to 570) in a study involving 100 Pinnacle shells (DePuy, Warsaw, IN, USA) inserted into 21 patients (eight men and 13 women). Jin et al[[6]] reported a mean deformation of $65 \mu \mathrm{m}$ (25 to 103) in seven custom-made acetabular components inserted into cadavers. Liu et al[[8]] reported a mean deformation of $41.7 \mu \mathrm{m}$ (SD 8.9) in six Durom (Zimmer Inc, Warsaw, IN, USA) acetabular components inserted into surgically-prepared cadaveric pelves. The maximum deformation in our study (340 $\mu \mathrm{m})$ could be sufficient to interfere with the assembly process of a modular acetabular system. The clearance of such modular systems is likely to be of the order of $80 \mu \mathrm{m}$ and $120 \mu \mathrm{m}$.[[6]]

In all cases the surgeon was able to obtain a satisfactory press-fit, despite the variability in the strength of the bone. Figure 3 shows a potential relationship between the surgeons's grading and his/her ability to identify the softest and hardest bone correctly. However, the mid-range bone was more difficult to assess accurately. Owing to the small sample size, no statistical analysis was performed.

There were several limitations to the study. First, during the compression tests, 13 samples of bone toppled over during loading, rather than failing via compression. This was probably caused by the ends not being trimmed perpendicular to the axis of the cylinder of 
bone. In order to overcome this obstacle, the results for the left and right femora were combined and the peak modulus and yield stresses compared with deformation. The second main limitation was the risk of microfractures by implanting multiple acetabular shells in one acetabulum. To reduce the risk of this the surgeon would inspect the acetabulum after removing a shell, to ensure that the bone was not visually fractured, before reaming and implanting the next shell. No visible fractures were identified by the surgeon in this study. The third limitation was the need to use different diameters of shell. Ideally all samples would have been of the same diameter, as it has been shown that this can influence the extent of deformation, but because of the limited numbers of cadavers available, this was not possible. However, the results of the statistical analysis showed no correlation between the diameter of the shell and deformation.

The final limitation was using the properties of the bone of the femoral head as a surrogate for that of the acetabulum. This was done because the reaming process leaves little bone available in this location. Also, the reaming process itself may have caused damage to the bone that would weaken it, if its strength were to be measured retrospectively.

We have identified only one study measuring the properties of bone around but not within the acetabulum.[[16]] This found relatively low values of Young's modulus in pelvic bone, rarely exceeding $100 \mathrm{MPa}$. Owing to its sandwich construction, with trabecular bone contained within a thin cortical shell, the overall mechanical behaviour of the pelvic bone has limited sensitivity to variation in the mechanical properties of trabecular bone.[[17]]

Given that these bones form opposing load-bearing regions, it is reasonable, in our view, to suppose that the bone may have similar properties on either side, as has been shown for the instantaneous modulus of articular cartilage over opposing joint surfaces.[[18]]

As deformation of the shell occurs predominantly as a result of the non-uniform acetabular structure, it is necessary to perform further research. It has previously been shown 
that the shape of the acetabulum results in two-point loading of uncemented acetabular shells. Therefore, such a study would involve determining how the strength of the bone of the femoral head relates to the stresses required to expand the acetabulum by a given value. This study has shown that the strength of the bone has no relationship with the amount of deformation of press-fit acetabular shells. This lack of association is possibly explained by the fact the surgeon achieved a satisfactory surgical press-fit despite the difference in bone quality. Further studies examining the relaxation of the bone are recommended to determine how the deformation of the shell develops over longer periods. 


\section{Author contributions}

M. C. Bone: Design of investigation, Data collection, Data analysis, Writing paper.

P. Dold: Design of investigation, Data collection, Data analysis, Paper review, Scientific discussion.

M. Flohr: Review analysis, Paper review, Scientific discussion.

R. Preuss: Paper review, Scientific discussion.

T. Joyce: Paper review, Scientific discussion.

R. M. Aspden: Data analysis and interpretation, Revising the paper.

J. Holland: Performed surgeries, Data collection. Co-writer/editor of manuscript.

D. Deehan: Designed study, Reviewed results, Performed surgery, Removed bone cores for testing.

The authors would like to thank N. Taylor (3D Measurement Company) for his work with regard to data acquisition and processing of experimental data. We would also like to thank Dr A. Blain of Newcastle University for performing the statistical analysis

The research was supported by the NIHR Newcastle Biomedical Research Centre.

The authors P. Dold, M. Flohr and R. Preuss are employed by Ceramtec

GmbH. Martin Bone received a salary from the joint fund.

The author or one or more of the authors have received or will receive benefits for personal or professional use from a commercial party related directly or indirectly to the subject of this article. 


\section{References}

1. Hailer NP, Garellick G, Kärrholm J. Uncemented and cemented primary total hip arthroplasty in the Swedish Hip Arthroplasty Register. Acta Orthop 2010;81:34-41.

2. No authors listed. National Joint Registry 10th Annual Report 2013. http://www.njrcentre.org.uk/njrcentre/default.aspx. (date last accessed 13 January 2015).[[bibmisc]]

3. Kwong LM, O’Connor DO, Sedlacek RC, et al. A quantitative in vitro assessment of fit and screw fixation on the stability of a cementless hemispherical acetabular component. $J$ Arthroplasty 1994;9:163-170.

4. Squire M, Griffin WL, Mason JB, Peindl RD, Odum S. Acetabular component deformation with press-fit fixation. J Arthroplasty 2006;21:72-77.

5. Elke R, Berli B, Wagner A, Morscher EW. Acetabular revision in total hip replacement with a press-fit cup. J Bone Joint Surg [Br] 2003;85-B:1114-1119.

6. Jin ZM, Meakins S, Morlock MM, et al. Deformation of press-fitted metallic resurfacing cups. Part 1: experimental simulation. Proc Inst Mech Eng H 2006;220:299-309.

7. Meding JB, Small SR, Jones ME, Berend ME, Ritter MA. Acetabular cup design influences deformational response in total hip arthroplasty. Clin Orthop Relat Res 2013;471:403-409.

8. Liu F, Chen Z, Gu Y, Wang Q, Cui W, Fan W. Deformation of the Durom acetabular component and its impact on tribology in a cadaveric model--a simulator study. PLoS One 2012;7:45786.

9. Goebel P, Kluess D, Wieding $\mathbf{J}$, et al. The influence of head diameter and wall thickness on deformations of metallic acetabular press-fit cups and UHMWPE liners: a finite element analysis. J Orthop Sci 2013;18:264-270. 
10. Yew A, Jin ZM, Donn A, Morlock MM, Isaac G. Deformation of press-fitted metallic resurfacing cups. Part 2: finite element simulation. Proc Inst Mech Eng H 2006;220:311-319. 11. Hothi HS, Busfield JJ, Shelton JC. Deformation of uncemented metal acetabular cups following impaction: experimental and finite element study. Comput Methods Biomech Biomed Engin 2014;17:1261-1274.

12. Dold P, Bone MC, Flohr M, et al. Validation of an optical system to measure acetabular shell deformation in cadavers. P I Mech Eng H 2014;228:781-786.

13. Bone MC, Dold P, Flohr M, et al. A novel method for measuring acetabular cup deformation in cadavers. Proc Inst Mech Eng H 2013;227:1341-1344.

14. Li B, Aspden RM. Composition and mechanical properties of cancellous bone from the femoral head of patients with osteoporosis or osteoarthritis. J Bone Miner Res 1997;12:641651.

15. Callister W Jr. Fundamentals of Materials Science and Engineering. Second ed. Wiley; 2005.

16. Dalstra M, Huiskes R, Odgaard A, van Erning L. Mechanical and textural properties of pelvic trabecular bone. J Biomech 1993;26:523-535.

17. Dalstra M, Huiskes R. The pelvic bone as a sandwich construction: a three dimensional finite elemet study [abstract]. ESB congress 1990.

18. Shepherd DE, Seedhom BB. The ‘instantaneous’ compressive modulus of human articular cartilage in joints of the lower limb. Rheumatology (Oxford) 1999;38:124-132. 